Time series of EIT chest images using singular value decomposition and Fourier transform

Kerrouche, $\mathrm{N}$ and McLeod, CN and Lionheart, WRB 2001

MIMS EPrint: 2015.37

Manchester Institute for Mathematical Sciences

School of Mathematics

The University of Manchester

\footnotetext{
Reports available from: http://eprints.maths.manchester.ac.uk/

And by contacting: The MIMS Secretary

School of Mathematics

The University of Manchester

Manchester, M13 9PL, UK
} 


\title{
TIME SERIES OF EIT CHEST IMAGES USING SINGULAR VALUE DECOMPOSITION AND FOURIER TRANSFORM.
}

\author{
N Kerrouche* , CN. McLeod*,W.R.B. Lionheart**. \\ * EIT Research Group, Oxford Brookes University, Oxford, UK. \\ ** Department of Mathematics UMIST, Manchester, UK. \\ nkerrouche@brookes.ac.uk
}

\begin{abstract}
The aim of this study is to propose a useful method for exploring regional ventilation and perfusion in the chest. The paper describes two methods based on Singular Value Decomposition (SVD) and Fourier Transform (FT) respectively. This work shows that Power Spectral Density (PSD) and phase images (derived from the Fourier transform) are easier to interpret and more useful tools for exploiting in vivo EIT data in healthy volunteers in order to explore the cardiovascular and respiratory systems.
\end{abstract}

\section{Introduction}

The EIT (1) reconstruction method inherently smoothes changes across the whole image making it difficult to resolve changes from different sources. The SVD method described below takes spatial and temporal data and analyses them together. In order to understand ventilation or perfusion in the lungs we have to re-integrate more than one principal time series and corresponding principal image. An alternative method is to treat each pixel of an image as independent and to analyse the conductivity of the pixel sequence using the Fourier Transform (FT).

Since the main impedance changes in the chest are cyclical, the FT will highlight the principal temporal changes. As long as physiological changes occur at different frequencies, the related conductivity changes can be observed as images of those Fourier components. Images of both magnitude and phase of any component can be made. The interpretation will still be subjective since there are no independent data to verify the measurements.

\section{Materials and Method}

The data being analysed in this presentation were obtained from an Electrical Impedance Tomograph (OXBACT-3) [1] comprising 32 independently programmable current sources and 32 voltage measurement channels attached respectively to separate electrodes around the chest of a male volunteer. The 64 (ECG) electrodes were equally spaced on the skin in a saggital plane approximately $2 \mathrm{cms}$. below the level of the nipples. Each image was obtained by applying -in sequence- the first 10 spatial trigonometric current patterns. The $40 \mathrm{kHz}$ excitation current was used, a frequency at which a maximum value of $1 \mathrm{~mA}$ could be applied safely. Voltages were measured on each channel to a resolution exceeding 14 bits.

Two sequences of images were made, the first during normal breathing at a frame rate of 15 per second; 160 images cover two and a half breaths. The second sequence was made while the subject held his breath, thus removing the respiratory element from the data and allowing the cardiacsynchronous component to be more obvious. Independent respiratory and cardiovascular measurements were made with a 'Respitrace' monitor (Studley Data Systems, Oxford) and an Ohmeda 'Finapres' monitor respectively. These monitors provide only gross timing information of the cycles; 
there is no simple means of ascertaining regional ventilation or perfusion data which could be related to changes which might appear in the EIT data.

The reconstruction problem for EIT is non-linear and highly ill-posed [2]. Consequently the reconstructed images are at best highly smoothed pictures of the true conductivity. Current also strays out of the plane of the electrodes but the images were reconstructed on a two dimensional model.

Each image sequence consisted of $N=160$ images represented on a $M=450$ finite element mesh adapted to the subject's body shape. The (temporal) mean is removed from each image and these are assembled into a $M \times N$ matrix A.

\section{2-1. The singular value decomposition}

The singular value decomposition of the matrix A is calculated (using MATLAB 5)

\section{$\mathbf{U} \Lambda \mathbf{V}^{\mathbf{T}}=\mathbf{A}$}

where $\mathbf{U}$ is a $M \times M$ orthogonal matrix whose columns $\mathbf{u}_{i}$ we refer to as the principal images, (left singular vectors) $\mathbf{V}$ is a $N \times N$ orthogonal matrix of principal time series (right singular vectors) $\mathbf{v}_{i}$ and $\Lambda$ is a diagonal matrix of positive decreasing singular values $\lambda_{1} \geq \lambda_{2} \geq \cdots \lambda_{\min (M, N)} \geq 0$ augmented by rows or columns of zeros to make it an $M \times N$ matrix. The correlation matrix for the time series of nodal conductivities is $\frac{1}{N^{2}} \mathbf{A}^{T} \mathbf{A}=\frac{1}{N^{2}} \mathbf{U}^{T} \Lambda^{2} \mathbf{U}$ and the columns of $\mathbf{U}$ the principal components. However in contrast to the case where our data consists of samples from a vector of random variables we have a vector of time series. In this case the right singular vectors also carry useful information.

Let us define $\mathbf{A}_{k}=\mathbf{U} \Lambda_{k} \mathbf{V}^{\mathbf{T}}$ where $\Lambda_{k}$ is the diagonal matrix with diagonal $\lambda_{1}, \lambda_{2}, \ldots \lambda_{k}, 0, \ldots$. This corresponds to truncating the singular value decomposition. Defining the matrix norm $\|\mathbf{A}\|^{2}=\frac{1}{N^{2} M^{2}} \sum_{j=1}^{N} \sum_{i=1}^{M} a_{i j}^{2}$ we have the error due to this truncation is $\left\|\mathbf{A}-\mathbf{A}_{k}\right\|^{2}=\frac{1}{N^{2} M^{2}} \sum_{i=1}^{\min (M, N)} \lambda_{i}^{2}$. If each nodal value is known only with accuracy $\boldsymbol{\varepsilon}$ then we can justify this truncation if $\left\|\mathbf{A}-\mathbf{A}_{k}\right\|^{2}<\varepsilon^{2}$. It is customary to refer to the first $k$ principal components explaining a proportion

$\sum_{i=1}^{k} \lambda_{i}^{2} / \sum_{i=1}^{\min (M, N)} \lambda_{i}^{2}$

of the total variance.

If for example we can only justify including the first principal component, that is $k=1$, then we can regard the entire time series of images as being one image $\mathbf{u}_{1}$ with each nodal value multiplied by the time series $\mathbf{v}_{1}$ scaled by $\lambda_{1}$. Instead of having $M N$ items of data we have in fact only $M+N+1$ which are significant.

\section{2-2 The Fourier transform}

From the EIT reconstruction algorithm the following outputs are available:-

1. Reconstructed raw EIT image,

2. Time series for each pixel, 
Then the power spectral density (PSD) and phase of the matrix A are calculated using the FFT function of MATLAB 5 to generate the following outputs:-

1. PSD per pixel

2. A magnitude image at each frequency

3. Phase spectrum per pixel

4. A phase image at each frequency.

\section{Results}

\section{3-1 Singular value decomposition}

The SVD is obtained using the command $[\mathrm{u}, \mathrm{s}, \mathrm{v}]=\operatorname{svd}(\mathrm{A})$ where:

$\mathrm{u}$ : left singular vectors (images),

$\mathrm{v}$ : right singular vectors (time series) and

s: decreasing singular values.

The choice of truncation level is somewhat subjective in this case as it is hard to estimate the error in the reconstructed images. There is a noticeable drop between the first and second singular values in both cases (they are plotted on a logarithmic scale in the figures).

\section{a) Breath Holding}

The breath holding image sequence shows clearly the thirteen cardiac cycles over this period (figure 2-3). The lungs are visible as low conductivity regions in the mean image (figure 5). The second principal component is at the cardiac frequency and by definition orthogonal to the first (figure $3)$. It can be seen to be out of phase.

The singular values are $\lambda_{\mathrm{i}}=0.0075,0.0033,0.0014,0.0011,0.0011,0.0008 \ldots$.

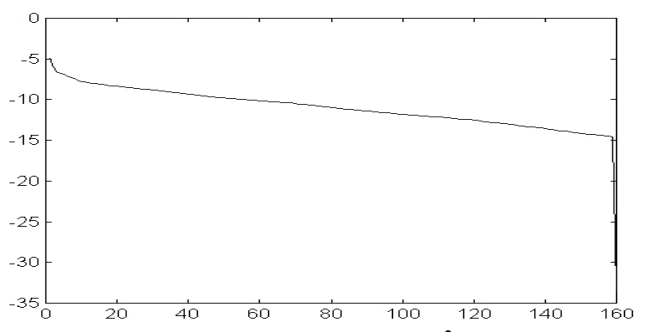

Figure1: Singular values $\log _{10} \lambda_{i}$

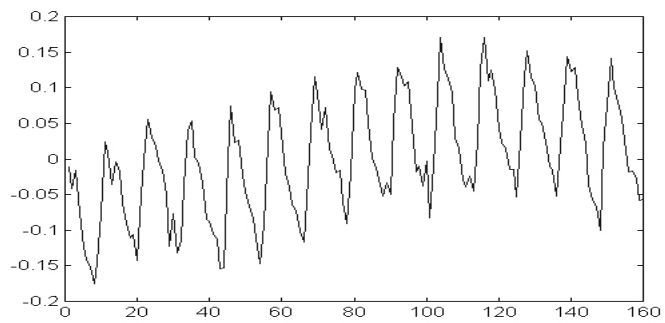

Figure 3: Principal time series $\mathbf{v}_{2}$

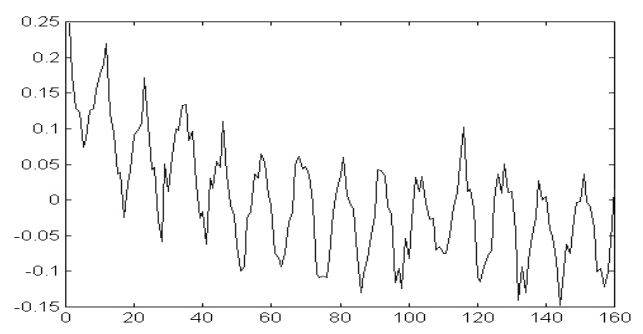

Figure 2: Principal time series $\mathbf{v}_{1}$

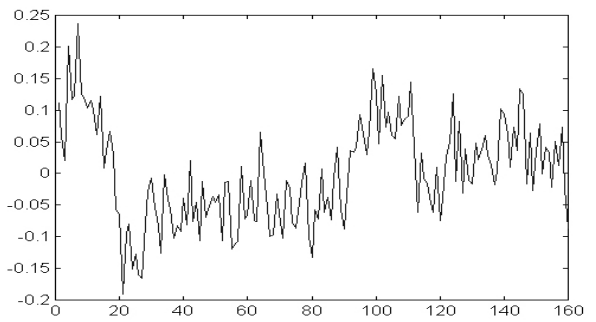

Figure 4: Principal time series $\mathbf{v}_{3}$ 

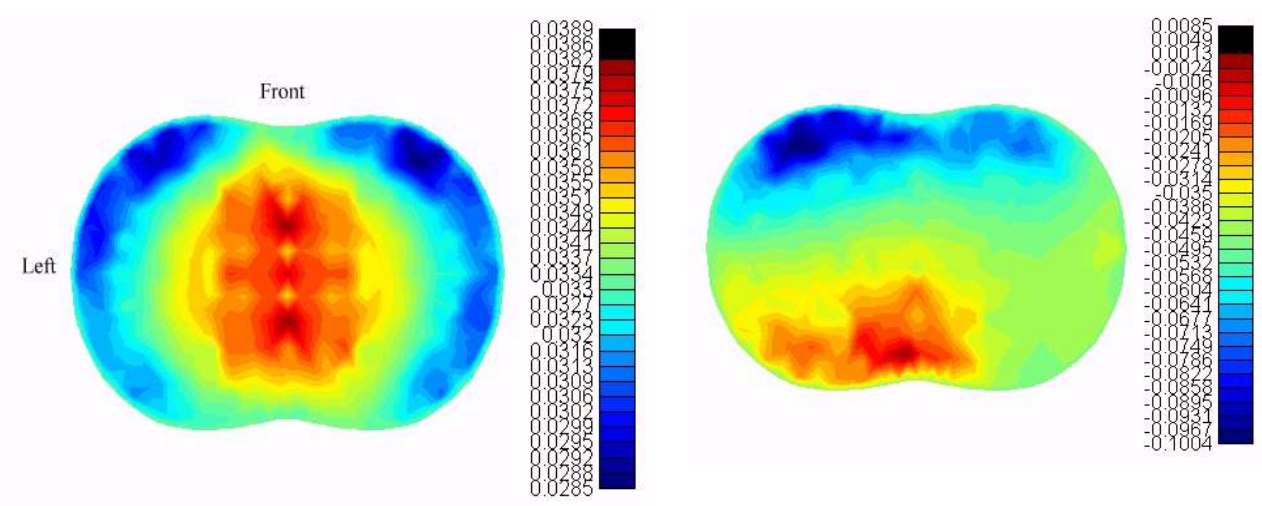

Figure 5: Temporal mean image

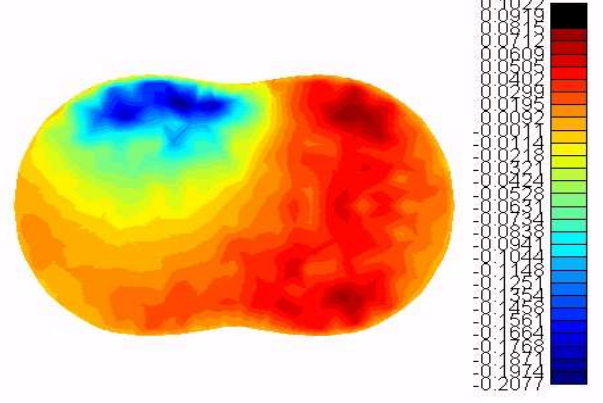

Figure 6: Principal image $\mathbf{u}_{1}$

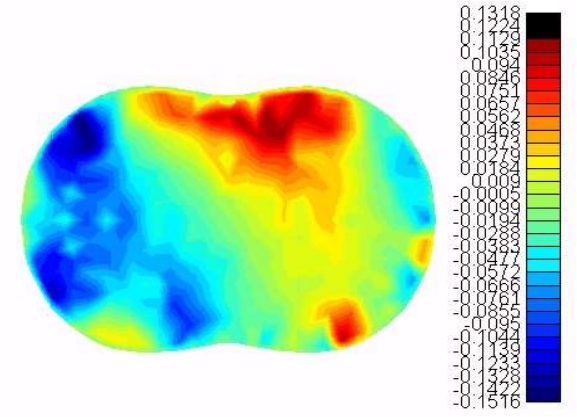

Figure 7: Principal image $\mathbf{u}_{2}$

Figure 8: Principal image $\mathbf{u}_{3}$

\section{b) Breathing}

The breathing study has a similar mean (figure 13), but this time the first principal time series follows the respiratory cycle (figure 10). The first principal image (figure 14) indicates that both lungs change conductivity simultaneously. The second principal time series (figure 11) is mainly the first harmonic of the respiratory cycle but also includes a significant cardiac frequency component. However, note that $\lambda_{2}$ is $\cong 1 / 40 \lambda_{1}$.

The singular values are $\lambda_{\mathrm{i}}=0.3421,0.0091,0.0054,0.0037, \ldots$

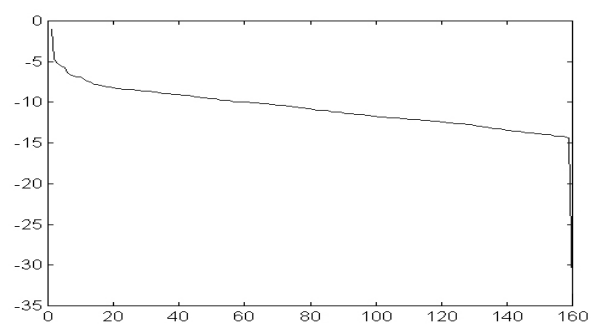

Figure 9: Singular values $\log _{10} \lambda_{i}$

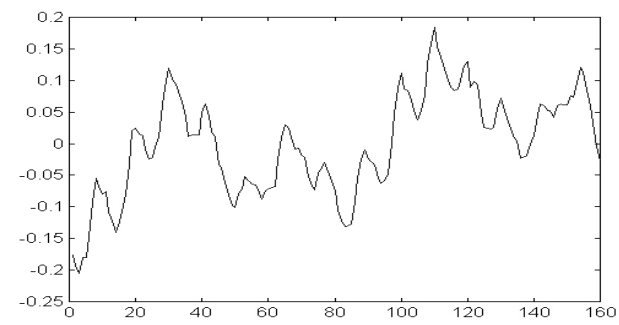

Figure 11: Principal time series $\mathbf{v}_{2}$

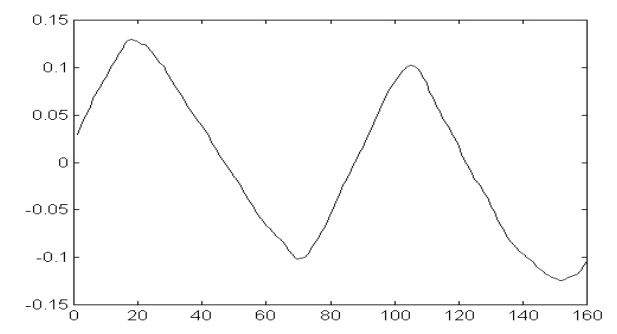

Figure 10: Principal time series $\mathbf{v}_{1}$

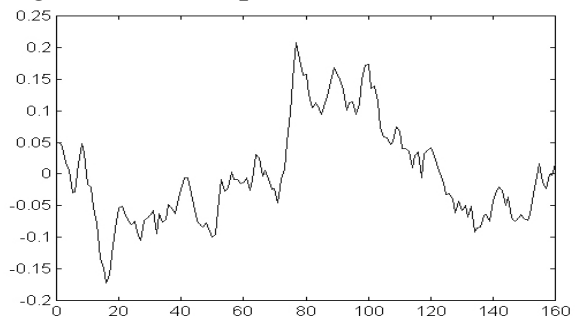

Figure 12: Principal time series $\mathbf{v}_{3}$ 


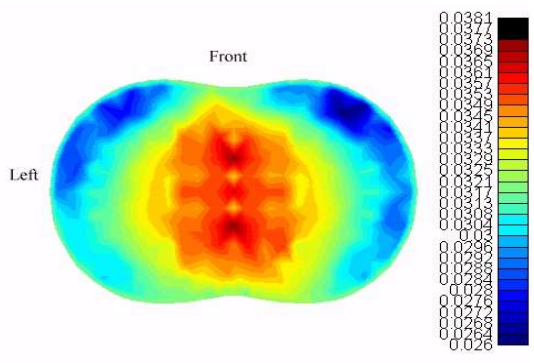

Figure 13: Temporal mean image

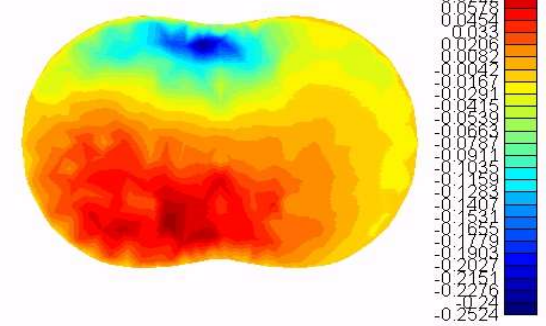

Figure 15: Principal image $\mathbf{u}_{2}$

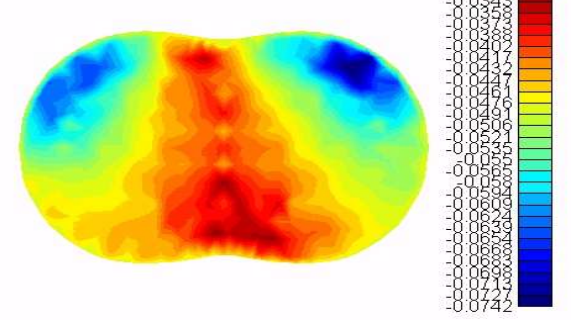

Figure 14: Principal image $\mathbf{u}_{1}$

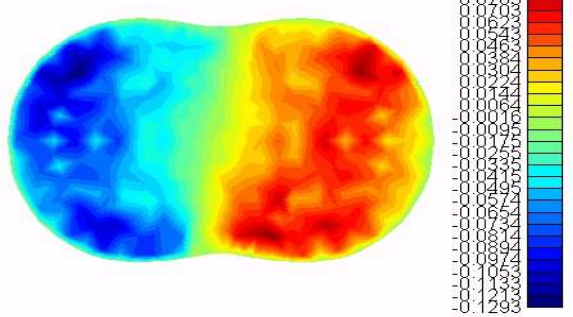

Figure 16: Principal image $\mathbf{u}_{3}$

\section{3-2 Fourier Transform}

\section{a) Breath Holding}

The time series of a heart pixel shows clearly the cardiac component (figure 17-18). However a lung pixel shows a slow component greater than the component at the heart frequency (figure 19-20). At the heart frequency there is a phase difference between the region of the heart and the rest of the chest (figure 22)

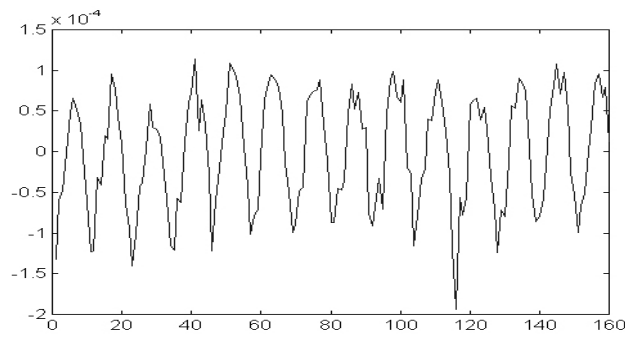

Figure 17: Time series of a heart pixel

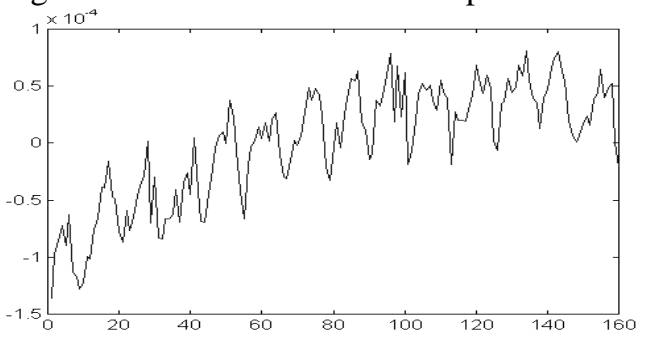

Figure 19: Time series of a lung pixel

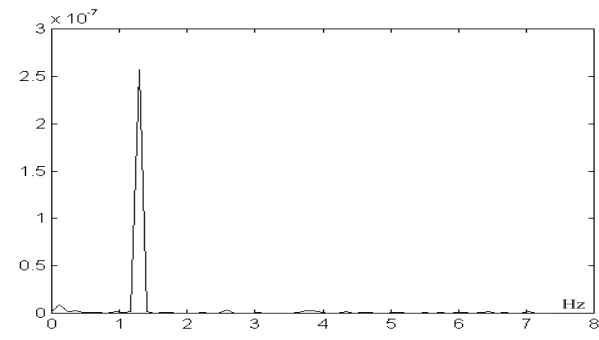

Figure 18: PSD of a heart pixel

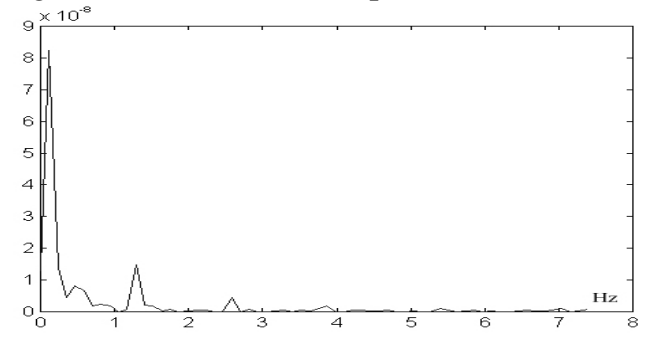

Figure 20: PSD of a lung pixel 


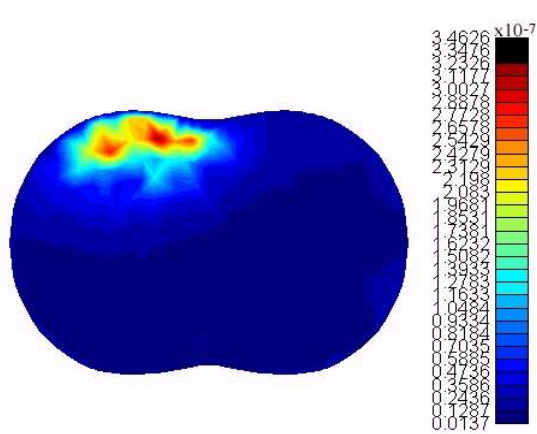

Figure 21: PSD at the heart frequency

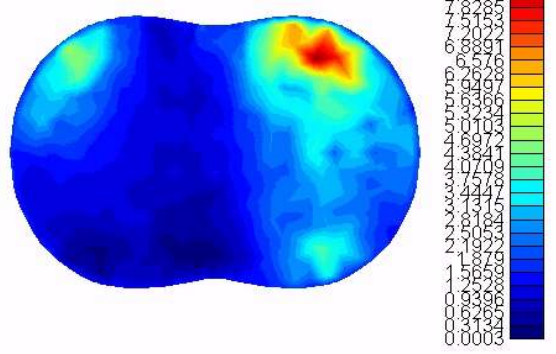

Figure 23:PSD at the low frequency

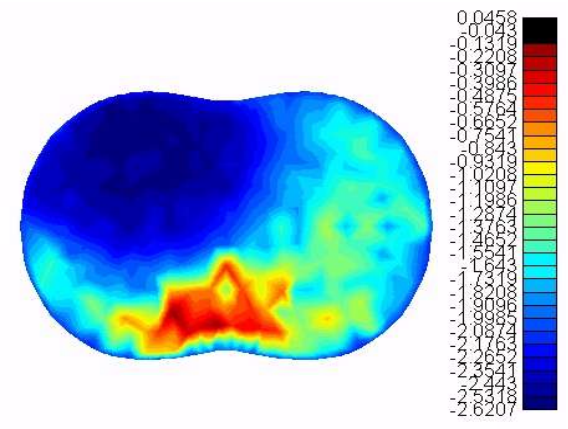

Figure 22: Phase at the heart frequency

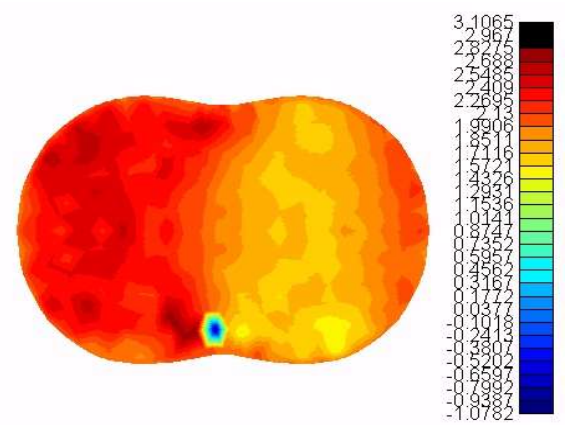

Figure 24:Phase at the low frequency

\section{b) Breathing}

The breathing sequence shows a very large harmonic peak at $0.2 \mathrm{~Hz}$-breathing component(figure 26) in every pixel with the maximum amplitude in the region of the lungs (figure 29) and a less significant heart component as well.

The conductivity of pixels taken from a lung and from the respitrace are as expected in anti phase (figure 25). The time series of a heart pixel shows changes due to the activity of the heart superimposed on changes due to breathing (figure 27). The breath holding sequence shows more clearly a heart related FFT peak at $1.28 \mathrm{~Hz}$ than the breathing sequence does (figure 18 referred to figure 28).

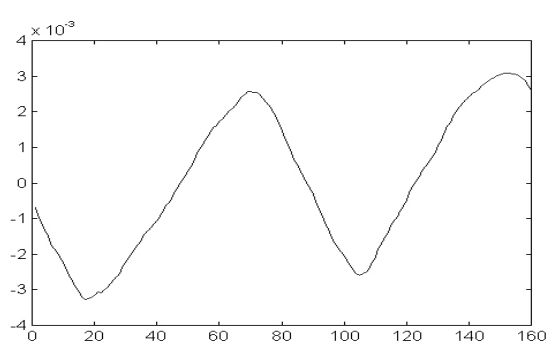

Figure 25: Time series of a lung pixel

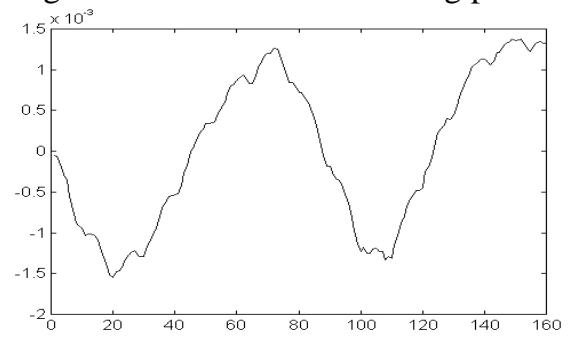

Figure 27: Time series of a heart pixel

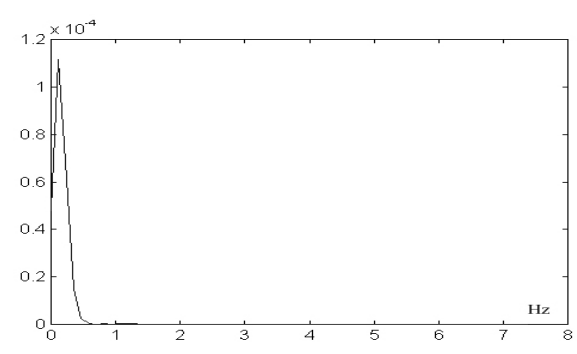

Figure 26: PSD of a lung pixel

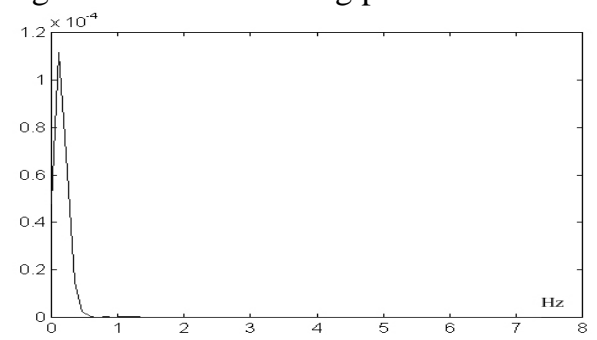

Figure 28: PSD of a heart pixel 


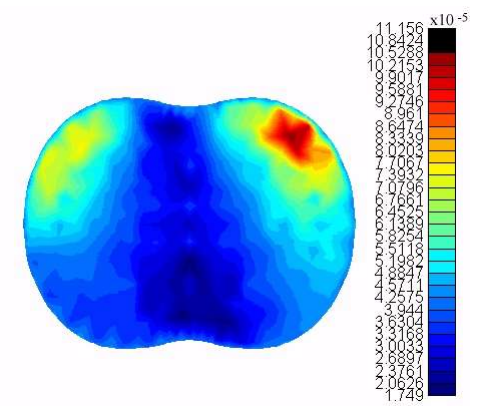

Figure 29: PSD at the breathing frequency

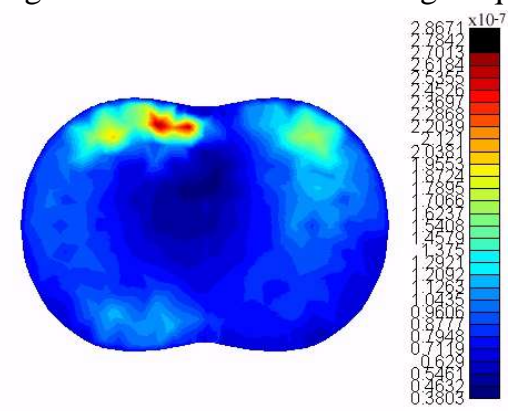

Figure 31: PSD at the heart frequency

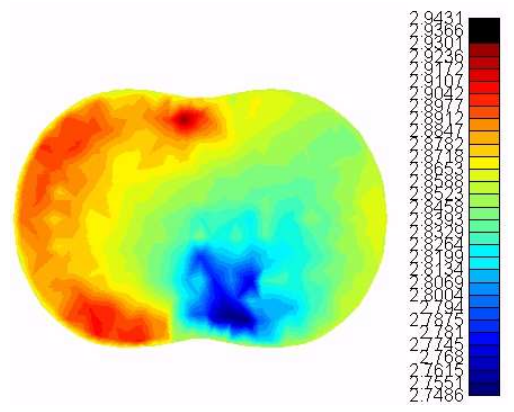

Figure30:Phase at the breathing frequency

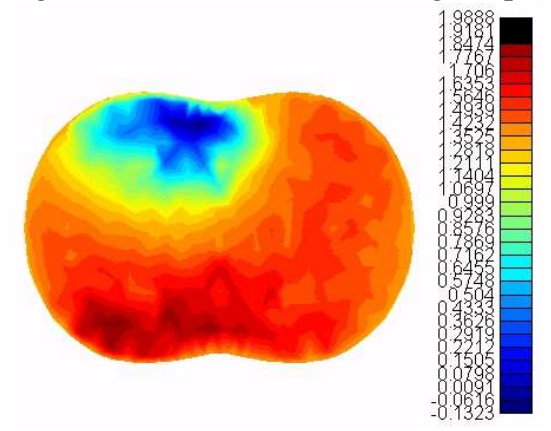

Figure 32: Phase at the heart frequency

\section{Discussion}

The interpretation of EIT chest images is a new skill, having, in common with ultrasound images and the occasionally performed X-ray fluoroscopy, to take account of both temporal and spatial information. Normal vision detects both, but the spatial resolution is much superior to the temporal resolution. An example is the comparison of the rotation speed of two objects which are not in the same field of view.

From raw images - which have relatively poor spatial definition- an observer can resolve large anatomical features and both respiratory- and cardiac- synchronous changes. The magnitudes of these changes in different parts of a sequence of images are hard to determine; the choice of colour or grey scale can improve the measurement but it is clear that objective processing of the whole data will improve understanding of the images.

\section{Interpretation of SVD results}

The principal components of the data are extracted as (a scale factor $\lambda$ ) $\mathrm{x}$ (a principal time series) $\mathrm{x}$ (a principal image) in a completely objective process. Each component will be orthogonal to all the others.

During breath holding, the first two principal time series are at the cardiac frequency and must be $90^{\circ}$ out of phase in order to be orthogonal. They represent cardiac synchronous changes in impedance at different phases and a full understanding of the temporal relationships in blood volume across the image would require a recombination of these two components. The third and subsequent singular values are much smaller. The random nature of the third time series suggests that noise is the main feature - electronic, experimental and reconstruction.

During breathing a very clear respiratory time series is seen in the first principal component. It shows values which are highest in the lung regions and non-zero where no breathing signal could be expected. This effect is inherent in the method as EIT smoothes regional changes. The first time series follows the Respitrace signal perfectly. Regional ventilation information can be obtained from the first principal image with some confidence as the scale of change on the key shows a ratio of about 1:1.5 inside the lungs and 1:2.5 from smoothing.

The second principal time series is approximately $1 / 40$ the size of the first and demonstrates that the SVD method is capable of extraction small components from noisy data. Both respiratory and cardiac 
synchronous changes are seen; no cardiac component was obvious in the raw animation. The largest values on this principal image are seen in the region of the heart. However, the respiratory and cardiac contributions are not separable in this method.

The third principal image shows some left-right changes with timing at some non-respiratory frequency. It is possible that this an experimental artefact; the low level of the changes allows little confidence in its interpretation.

\section{Interpretation of FT results}

During breath holding the most significant temporal change is seen over the whole image at the heart frequency. Lung conductivity is a function of the local blood volume. The PSD of the lung pixel time series shows two components, one very slow and the second at the heart frequency. The first may be related to the change in intra-thoracic pressure at the onset of breath holding. This aspect of the experimental protocol was not controlled. The second component at the heart frequency, can be examined in the images of PSD and phase. Here it is clear that the largest PSD is around the heart - the amplitude being more than 100 times the amplitude in the lung region. The phase image shows that the cardiac synchronous blood volume changes in the heart are more than $90^{\circ}$ out of phase with much of the lungs. Magnitude and phase information must be taken together to understand the blood volume changes. Independent verification of the timing of lung tissue blood volume changes in humans is unavailable.

During breathing a clear respiratory time series is seen on both lung and heart pixels. As mentioned, the EIT method smoothes changes across the image. By examining the magnitude and the phase at that frequency alone it can be seen that the magnitude of change in the lungs is several times larger than in other regions, and that regional ventilation differences in the lungs can be seen. The phase image is almost uniform $\left(<10^{\circ}\right.$ over the image).

By selecting the heart frequency, the image of cardiac-synchronous changes can be extracted. The magnitude shows a 5:1 ratio between the change in the heart and outside it. The phase information is resolvable and shows typically $90^{\circ}$ difference between the heart and lung regions.

\section{Conclusion}

Raw EIT images demonstrate respiratory and cardiac related conductivity changes. SVD and FT analyses of these changes has enabled the separation of different components and demonstrated changes which would be expected in normal physiology. The SVD method integrates spatial and temporal changes in its analysis which makes its outputs more complex to interpret. The FT method is sensitive only to temporal changes and therefore is more appropriate where the region being imaged has parts which stay in place. The extra precision offered by the FT has been shown to enhance the diagnostic power of the EIT method when used to study physiological changes in the thorax.

Acknowledgement: This work is supported by the Wellcome Trust.

\section{References}

[1] Zhu Q, et al, Development of a real-time adaptive current tomograph, Physiol Meas V 15 Suppl 2A 37-45 1994

[2] Breckon W.R \& Pidcock M.K., Ill-posedness and non-linearity in electrical impedance tomography, In Information processing in Medical Imaging, Eds de Graaf \& Viergever, Plenum, 235244,1987 